\title{
¿Cómo el interés y la motivación influyen en el aprendizaje de una lengua extranjera?
}

\author{
Clara Esperanza Bilbao ${ }^{1}$
}

Recibido: 15-08-2017

Aceptado: 03-11-2017

\section{RESUMEN}

Aprender una lengua extranjera puede ser para algunas personas una experiencia interesante, fácil y productiva pero para otras; aburridora, complicada e incluso inútil. Sin embargo, es un hecho que en la mayoría de los casos, independientemente de la percepción que se tenga, el estudiante no alcanza la misma competencia en la lengua extranjera que en la lengua materna.

Aunque este hecho se puede explicar a través de factores específicos al proceso de adquisición de la lengua (los cuales son muy decisivos), también podemos hallar respuesta en factores relacionados con el entorno del estudiante, que determinan desde el momento en que se aprende la lengua, ciertas actitudes y motivación que favorecerán o no la adquisición y el posterior nivel de competencia en ésta.

Palabras clave: Lengua extranjera, lengua materna, aprendizaje, actitud, motivación. 


\title{
How do interest and motivation affect foreign languages learning?
}

\begin{abstract}
To learn a foreign language can become, for some people, an interesting and easy experience but for others, a worrying and complicated one. However, in most of the situations the student can not get the same competeces in a foreign language as he/she did it in his/her first language.

Although it can be explained through specific factors related to language acquisition process, we can also find answer in factors related to the student environment which define, since the beginning, some attitudes and motivation which will favor or not the language acquisition and the competences he/she has.
\end{abstract}

Keywords: Foreign language, first language, learning, attitude, motivation.

Antes de iniciar, deseo aclarar el significado de las siglas L1 y L2, las cuales utilizaré con frecuencia a lo largo del texto: L1 hace referencia a la lengua materna o primera lengua que adquirimos desde que nacemos y $L 2$ a la(s) lengua(s) extranjera(s) que aprendemos a lo largo de nuestra vida. Asimismo, usaré la sigla LE que significa "lengua extranjera".

Considero importante recalcar que todo proceso de aprendizaje se ve condicionado por dos elementos importantísimos: actitud y motivación, los cuales definiré a continuación para tener en claro su semejanza y similitud y así identificarlos más fácilmente.

Las actitudes según D. Backman y P. Secord (1971) son "el mecanismo individual a través del cual las influencias biosociales y socioculturales se traducen en la conducta manifiesta de un individuo. Las actitudes de una 
persona influyen en sus comportamientos de acercamiento o evasión hacia el mundo exterior".

En cuanto a la motivación, A. Maillo indica en la Enciclopedia Didáctica Aplicada la siguiente definición "la palabra motivación viene etimológicamente del vocablo latino "motio" que significa "movimiento". Un motivo es algo que empuja e incita a la acción. Una actitud es semejante a un motivo, por cuanto se refiere a la dirección de la conducta y no a la conducta en sí misma. El motivo aparece, desaparece y reaparece pero la actitud persiste (...). Las actitudes representan así, orientaciones generales persistentes del individuo frente a su medio, mientras que los motivos representan orientaciones que son temporales y relativamente especificas".

Teniendo en cuenta los conceptos y las definiciones anteriormente mencionados, empezaré por hablar de los factores ligados al entorno o ambiente psicosocial del estudiante y dejaré los específicos para más adelante.

Como resultado de muchas investigaciones, M. de Greve (1971) precisó los siguientes como los factores más influyentes en la motivación hacia el aprendizaje de una lengua extranjera: la familia, el profesor, los intereses y necesidades del estudiante. Ellos son los responsables, según de Greve, de que el estudiante considere importante o no aprenderla. En este punto pienso que, aunque hay muchos más factores que afectan la competencia que podemos adquirir en otra lengua, es verdad que el punto inicial de todo proceso de aprendizaje es ver la utilidad y necesidad de lo que vamos a aprender. Si estudiamos por obligación o porque "nos toca", no somos tan receptivos y adquisitivos como cuando lo hacemos por gusto, lo vamos a poner en práctica y sabemos que esa nueva área de conocimiento aportará algo a nuestra vida personal, profesional y/o laboral.

Pienso que cuando algo no nos parece importante para aprender, es como si cerráramos una puerta interna que bloquea nuestra receptividad y asimilación de lo que nos ofrecen y esto desemboca en no progresar mucho en el proceso. En el caso especifico de una lengua extranjera, obtenemos como resultado un bajo nivel de competencia y eso me lo ha dado la experiencia como docente de esta área.

Vale la pena citar aquí una idea de A. Aguayo (1947) con la que estoy plenamente de acuerdo y que tiene estrecha relación con lo planteado 
anteriormente: "el auténtico aprendizaje sólo se verifica cuando el estudiante está realmente interesado, motivado y empeñado en aprender. Sólo aprendemos aquello que corresponde a una necesidad, a un interés y a un ideal que se afirma en nuestra conciencia".

Veamos algunos de ellos más claramente a través de ejemplos: tomemos el caso de un estudiante de secundaria tipo A, con buenos recursos económicos $y$, por lo tanto, con muchas posibilidades de seguir una carrera profesional y salir del país, bien sea en plan de turismo, estudio y/o trabajo. Muy seguramente, este estudiante se desenvuelve en un medio social en el que sus padres, amigos y familia hablan otros idiomas y ya han viajado a algún destino internacional. Por consiguiente, para este estudiante será vital el aprendizaje de la lengua extranjera pues le ve una necesidad y aplicabilidad real y por lo tanto, estará motivado y con buena actitud hacia el aprendizaje, lo que favorecerá el logro de buenos resultados académicos en la asignatura y un alto nivel de competencia en la lengua.

Por el contrario, aquel estudiante de clase social baja tipo B, para el cual la culminación de sus estudios de colegio significará el inicio de una vida laboral para ayudar a sostener económicamente a su familia ya que sus padres cuentan con serios problemas económicos para subsistir y poder educar también a sus hermanos; verá muy lejana la oportunidad de formarse profesionalmente y/o salir del país, por lo que considerará el aprendizaje de la lengua como un espacio tal vez interesante pero poco útil para su vida futura. Esto se reflejará, sin duda, en una actitud de desmotivación y desinterés en clase.

La definición de "motivación" dada por T. Risk (1964) encaja, perfectamente, en lo que acabo de ejemplificar: "la motivación es el desarrollo en los alumnos de un interés y una favorable actitud hacia las actividades que debe dar por resultado un aprendizaje eficaz. Si al alumno no se le despierta el interés por aprender, no va a rendir de la misma manera que otro que tenga objetivos claros para su aprendizaje".

Paso ahora a tratar los factores que están más ligados a la parte psicolingüística, sociolingüística e interactiva que influyen, de forma decisiva, en la adquisición de una lengua extranjera y en la competencia que tenemos en ella.

Para iniciar, me parece importante que nos cuestionemos sobre qué entendemos por ser "bilingüe". Hay quienes dicen que ser "bilingüe" 
significa poseer conocimientos en más de una lengua pero no lo creo así ya que el hecho de que alguien tenga interiorizada una serie de conceptos, reglas y estructuras no quiere decir que las pueda utilizar correctamente si lo exponemos a una conversación, redacción de un documento o interpretación de una lectura. Encontré entre algunos aportes de C. Atlasovich (experta en estudios de neuropsicología aplicados a la adquisición bilingüe) una definición, veamos: "ser bilingüe es tener la habilidad para comunicarse efectivamente en dos o más lenguas, con el mismo grado de facilidad y competencia". Ella supera la definición común que mencioné inicialmente ya que usa las palabras "habilidad" y "comunicarse" y con esto se aproxima a mi idea pero hay algo en la parte final de su definición con la que no estoy muy de acuerdo porque no pienso que toda persona bilingüe posea el mismo grado de competencia en las dos lenguas que habla, a menos que las haya desarrollado en circunstancias lingüísticas especiales tales como por ejemplo, vivir en el país de la LE y a la vez estudiar y/o trabajar en el medio de la LE.

Adicionalmente, ella menciona dos conceptos bastante interesantes y que, en lo personal, no conocía: "Bilingüismo Balanceado" para referirse a cuando el individuo posee, aproximadamente, la misma fluidez en ambas lenguas y "Semilingüalismo" a cuando el individuo posee deficiencias en ambas lenguas comparadas con el hablante monolingüe. Así mismo, agrega que "según el momento de exposición a ambas lenguas, L1 y L2, se habla de dos tipos de adquisición del lenguaje en el bilingüe: "Adquisición simultánea" cuando el niño adquiere dos o más lenguas antes de los 3 años, es decir, recibe L1 y L2 desde el nacimiento y "Adquisición secuencial" cuando la L2 se introduce una vez que el niño ha adquirido y consolidado la L1, aproximadamente a los 3 años. Creo que esta clasificación de las adquisiciones reafirma mi punto de vista.

Al retomar la definición del "bilingüismo balanceado" hay algo que llamó bastante mi atención: "el individuo posee aproximadamente la misma fluidez en ambas lenguas" y lo que me genera en este momento una confusión de ideas pues $C$. Atlasovich mencionó en su definición de persona "bilingüe" que es quien maneja las dos lenguas con el mismo grado de facilidad y competencia y luego, en el "bilingüismo balanceado" afirma que el hablante bilingüe posee aproximadamente la misma fluidez en ambas lenguas. Es decir, finalmente, según ella, la persona bilingüe posee la misma competencia en L1 y L2? o no? Yo continuo pensando que la respuesta es no, a excepción de casos 
especiales en que la persona ha estado expuesta simultáneamente a las dos lenguas en ambientes naturales.

Entremos entonces a ver los elementos que, luego de una larga lectura e investigación, considero que influyen en la disparidad de competencias en la L1 y la L2.

Al interior de una clase de lengua extranjera, vemos, con frecuencia, un fenómeno en el cual aunque un cierto grupo de personas se encuentran aprendiendo la misma LE y bajo las mismas condiciones, el desempeño es tan variado como las mismas personalidades de cada uno de ellos. En su intento por comunicarse en el otro idioma, el estudiante termina por no hablar ni su L1 ni la L2 sino una especie de lengua hibrida conformada por elementos lingüísticos de las dos lenguas y esta combinación lingüística es lo que conocemos con el nombre de interlengua.

Según esta, al parecer, el estudiante refleja una competencia lingüística en evolución que se reorganiza durante el proceso de aprendizaje, aproximándose poco a poco a la competencia lingüística de los hablantes nativos de la LE.

El concepto de interlengua fue introducido por Selinker quien la definió como un estadio del proceso de aprendizaje que es independiente de la L1 y la L2. Antiguamente, se pensaba que la lengua materna influía de manera negativa en el aprendizaje de la otra lengua ocasionando la llamada transferencia lingüística. Sin embargo, hacia los años 70, se realizaron estudios en los que Krashen afirmó que las interlenguas presentan un orden de adquisición y Adams, que hay unas secuencias de desarrollo comunes.

De igual forma, algunos estudios han revelado, en el caso especifico de los errores de pronunciación en la lengua que se aprende, que muy pocos de ellos se deben a una transferencia lingüística directa de la lengua materna (lo que contradeciría las hipótesis antiguas) y que su causa real es el sistema lingüístico intermedio propio de su aprendizaje o interlengua. A este respecto, no estoy muy de acuerdo pues gracias a mi experiencia como docente de lengua extranjera he visto que los estudiantes muchas veces tienen interferencias en la LE a causa de sus estructuras de su L1 pues asumen que las dos funcionan de la misma manera. La interlengua, como podemos verlo, crea una interferencia lingüística que mientras no se supere, evita el alcance de una buena competencia en la L2. 
Por su parte, las teorías nativistas afirman que las lenguas se adquieren gracias a una condición innata inherente a la persona. Dentro de estas teorías, encontramos la Teoría de la Hipótesis Universal (la que pretende aplicar la teoría lingüística del generativismo a la adquisición de lenguas) y la Teoría del Monitor, formulada por Krashen y la cual se basa en 5 hipótesis que son: la de adquisición / aprendizaje, la del monitor, la del orden natural, la del input comprensivo y la del filtro afectivo.

La hipótesis de adquisición / aprendizaje distingue entre el aprendizaje en un entorno "natural" (contexto real de la LE, muy parecido al proceso de la lengua materna) y en uno "artificial" (aula de clase en entidades educativas). En esta hipótesis se afirma que la "adquisición" es lo que realmente queda en la persona y por lo tanto, permanece, mientras que el "aprendizaje" es temporal y tiene como función la de monitorizar la adquisición de la lengua. En el marco de esta afirmación, he comprendido que la $L_{1}$ se adquiere mientras que la $L 2$ se aprende y luego se adquiere, lo que quiere decir que las dos lenguas no pasan exactamente por el mismo proceso y que cuando aprendemos nuestra lengua materna, todo "se queda" pues hace parte del bagaje cognoscitivo, lingüístico, cultural, etc. que necesitaremos para desenvolvernos e interactuar con nuestro entorno natural. Por el contrario, cuando aprendemos una L2, solo parte del input permanece y es aquello que utilizamos para comunicarnos de manera adecuada. Con este análisis, se responde a la pregunta de por qué no obtenemos el mismo nivel lingüístico en L1 y L2.

Por otra parte, la hipótesis del monitor afirma que el estudiante tiene la capacidad de controlar, conscientemente, la forma en que se desenvuelve en la lengua extranjera. El uso adecuado del monitor consiste en tener un equilibrio entre la corrección y el cumplimiento de los objetivos comunicativos. Esta hipótesis confirma el hecho de que siempre que usamos nuestra lengua materna, lo hacemos de manera inconsciente, los procesos cerebrales que dan pie a lo que decimos, entendemos, leemos y escribimos surgen de forma natural, nada es premeditado y preparado como si lo es en la L2.

La hipótesis del orden natural considera que la lengua de adquiere en un orden predecible, lo cual se consigue gracias a mensajes y/o estímulos asimilables que es lo que conocemos con el nombre de input comprensible. En efecto, la adquisición no es producto de la enseñanza sino es el resultado natural a un estimulo. 
Un punto negativo que veo en esta hipótesis es que no considera las diferencias individuales en el aprendizaje, aspecto que si tiene en cuenta la hipótesis del filtro afectivo. Por esta razón, estoy de acuerdo con Krashen ya que según él, la autoconfianza, la motivación y el miedo, entre otros, son algunos de los factores psicológicos que pueden afectar significativamente el uso adecuado del estimulo recibido.

A pesar de las innumerables críticas que ha recibido la Teoría del Monitor, considero que hizo un gran aporte al dar pie a reflexiones sobre la diferencia aprendizaje / adquisición y el hecho de tener en cuenta la influencia del aspecto emocional del estudiante sobre su aprendizaje ya que, sin duda, la afectividad es uno de los elementos definitivos en el éxito o fracaso del proceso.

Si partimos del hecho de que la adquisición de las lenguas es algo innato, podríamos hablar de la existencia de una "gramática universal" cuyos principios generales son innatos, comunes y aplicables a todas las lenguas (universales lingüísticos) y lo cual significaría que a nivel gramatical, deberíamos ser capaces de asimilar fácilmente muchas de las reglas de la lengua que aprendemos sin ningún problema y adquirir un nivel similar al del hablante nativo pero no es así.

Sin embargo, las teorías cognitivas consideran el aprendizaje como un proceso complejo en el que el estudiante está limitado en la cantidad de input que puede procesar y en el que participan procesos cognitivos como la inferencia, la estructuración y reestructuración. Según estas teorías, las porciones de input que se asimilan se convierten en intake, lo que querría decir que no todo lo que recibimos se queda, lo cual nos pone en desventaja frente al nativo y explica, una vez más, por qué poseemos niveles de competencia diferentes en la L1 y la $L 2$.

Por su parte, a diferencia de la teoría del monitor y las teorías cognitivas, las cuales consideran que la condición innata es fundamental en la adquisición de una lengua, las teorías ambientalistas opinan que la experiencia o la educación son la base de la adquisición. Dentro de las ambientalistas encontramos la teoría de la aculturización y la de la acomodación.

En mi concepto, la aculturación no es otra cosa que el contacto entre dos culturas distintas (la de la lengua materna y la de la lengua extranjera). Sin embargo, C. Atlasovich añade otros factores que, según 
ella, afectan la adquisición de una lengua extranjera y que explican estas dos teorías: a) el fenómeno de aculturación o proceso de aprehensión de una determinada cultura, b) la actitud que se toma frente a la nueva cultura (si esta actitud es o no positiva, de respeto, deseo de aprender), c) la actitud del grupo inmigrante hacia el grupo dominante y/o a la inversa(discriminación o no), d) la personalidad del niño/adulto y e) los estilos de aprendizaje y estrategias para la enseñanza de la lengua extranjera.

No cabe duda de que todos estos factores sociales y culturales afectan desde el mismo instante del aprendizaje de la lengua extranjera, su adquisición y el desempeño que se tenga, posteriormente, en ella. Definitivamente, quien siente algún tipo de recelo (por algún tipo de experiencia negativa pasada) por los franceses, decide que Francia será el último lugar en el mundo al que quisiera ir y que no quiere tener ningún tipo de contacto con sus hablantes nativos, lo que termina en una oposición total a todo lo que tenga que ver con esa cultura (lengua, sociedad, literatura, turismo). Así, aunque la persona tuviera en algún momento de su vida, por fuerza mayor que aprender la lengua francesa, su "barrera" interna no permitirá que reciba, asimile, procese, aprenda y adquiera de forma ideal para alcanzar un nivel de lengua óptimo.

Aquí, entra en juego también el concepto de identidad ya que tiene que ver con el valor social que tenemos ante nosotros mismos y ante los demás y la lengua es, sin duda, uno de los "identificadores' de la identidad dentro de un grupo. Giles (1979) decía que en este rango se incluyen aspectos como el color de la piel, los rasgos faciales, la forma de vestir, las tradiciones culturales, los valores y también la lengua que se habla.

La identidad no es, sin embargo, un fenómeno estático. La teoría de la acomodación explica los ajustes a largo plazo en la pronunciación y otros aspectos del comportamiento lingüístico como un intento de aproximarse a la lengua de los hablantes nativos, si se consideran como deseables socialmente, o si el hablante quiere identificarse con ellos y/o demostrar buena voluntad hacia ellos, claro.

Con esto, podría decir entonces que, en relación con los grupos, el hablante no se identifica solamente con uno sino con varios al mismo tiempo. Mientras más compleja es una sociedad, más múltiples son las identidades. Encontré también las teorías interaccionistas que tienen en 
cuenta los factores innatos y ambientales para explicar cómo se produce la adquisición de una lengua.

Según Hatch (1978), quien propone la teoría del discurso, son las necesidades de comunicación las que nos incitan a desarrollar la lengua, trátese de la materna o de una extranjera. Esta teoría asume que el aprendizaje surge de la interacción con otros y, por lo tanto, que los factores externos tienen mayor importancia que los internos.

En el marco de la teoría del discurso, las características del input, las modificaciones interactivas y su efecto en la adquisición de una lengua, han sido uno de los factores que han despertado mayor interés y los considero totalmente pertinentes, por lo que los menciono enseguida.

Primero, tenemos el orden de adquisición o aprendizaje según el cual aunque todos tengamos una misma ruta en el proceso de aprendizaje, un estudiante de lengua extranjera jamás pasará por los mismos procesos que el que la aprende como lengua materna. Totalmente de acuerdo.

Segundo, cuando hablamos nuestra lengua materna, siempre tenemos una intuición sobre lo que es correcto y lo que no y eso de alguna forma nos orienta y nos ayuda a corregir pero cuando se trata de una lengua extranjera, no la poseemos y por eso encontramos una dificultad en el aprendizaje y tendemos a quedarnos estancados, lo que desemboca en que no avancemos en el aprendizaje. Este fenómeno es llamado fosilización. No conocía este término pero lo considero muy oportuno e interesante.

Tercero, el origen del input tiene mucha influencia en nuestro aprendizaje, por ejemplo, cuando aprendemos a hablar nuestra lengua materna, el input viene de nuestros padres, hermanos y toda persona cercana, en un cien por ciento del tiempo y en un ambiente natural.

Por el contrario, en el caso de una lengua extranjera, este viene de los profesores y compañeros de clase (también hablantes extranjeros de esta) y además de una forma limitada (solo durante la clase). Sin duda, este punto, al igual que los anteriores está ceñido a nuestra realidad y por eso, estoy de acuerdo en que este aspecto afecta nuestro proceso definitivamente. 
Cuarto, no es igual aprender una lengua cuando se tiene una necesidad comunicativa real ( $L 1)$ que cuando las necesidades son muy específicas y a veces temporales. En el colegio, por ejemplo, la necesidad es la de aprobar la asignatura; en la edad adulta, algunas veces la necesidad es la de aprender con el interés de un ascenso o un mejor salario.

De este documento se desprende que hay factores influyentes en el aprendizaje y adquisición de una lengua extranjera y que todos, sin importar su naturaleza, afectan el hecho de que nuestro nivel de competencia en una lengua extranjera no sea igual al de un hablante nativo. Sin embargo, es mucho lo que podemos hacer para que, a pesar de que el nivel de competencia no sea el superior, si haya una buena actitud hacia el aprendizaje y así, logremos que nuestros estudiantes realmente aprendan y sean capaces de comunicarse en la otra lengua.

De nosotros depende que los factores que influyan sean diferentes a los relacionados con la motivación ya que si ellos desean aprender la lengua y nosotros, como docentes, les damos las herramientas y las razones por las cuales es necesario e importante que lo hagan, el trabajo será más interesante y productivo.

De esta forma, considero necesario que como profesores reformulemos las metodologías y estrategias pedagógicas que no estén en coherencia con lo que demuestran las investigaciones. Es un hecho, que debemos empezar un trabajo investigativo en nuestras propias aulas, a través del cual conozcamos e identifiquemos mejor a nuestros estudiantes (quiénes son, qué quieren, qué piensan, qué necesitan). Como lo dijo Howard Hendricks "la enseñanza que deja huella no es la que se hace de cabeza a cabeza sino de corazón a corazón". 


\section{Referencias bibliográficas}

Aguayo, A. (1947). Pedagogía científica. Segunda ed. Habana Cultural.

Alcón, E. (2002). Bases lingüísticas y metodológicas para la enseñanza de la lengua inglesa. Publicacions de la Universitat Jaume. Recuperado el 14 de febrero de: http://books.google.com.co/books?id=gJuitmqCkpEC\&pg=PA67\&lpg=PA67\&dq=t eoria+del+monitor\&source=web\&ots=ZP9PidIKGI\&sig=Zcfib4vKpSh0t3Zh2DFB_1_ IQ8g\&h|=es\&sa=X\&oi=book_result\&resnum=5\&ct=result\#PPP1,M1

Backman, D. y Secord, P. (1991). Psicología social y educación, Buenos Aires.

Beltrán, L. (2015). Influencia cultural en la motivación para el aprendizaje del inglés como lengua extranjera. En Revista Interamericana de Investigación, Educación y Pedagogía. Volumen 8, No. 2.

Greve, M. (1971). Lingüística y enseñanza de lenguas extranjeras. Paris: Ed. Fragua.

Parra, H. y Romero, M. (1985). La actitud de los estudiantes de secundaria frente a la enseñanza del inglés. Universidad Nacional de Colombia: Tesis de grado.

Risk, T. (1964). Teoría y práctica de la enseñanza en las escuelas secundarias. México: Ed. Hispanoamericana.

Valdés, M. (2002). La vigencia del concepto de la Aculturación: alcances y limitaciones. Recuperado el 5 de Febrero de: http://www.mapuche.info/mapuint/valdes020500.

html

Vásquez, C., Romero, A. y Molina, R. (2009). Estrategias Pedagógicas para la Motivación Lectora a Través de un Software de Lectura Interactiva. En Revista Interamericana de Investigación, Educación y Pedagogía. Volumen 2, No. 2. 